\title{
Correspondence
}

\section{COMMENT ON MATERNAL ALCOHOL CONSUMPTION-RE: “CRYPTORCHIDISM AND HYPOSPADIAS AS A SIGN OF TESTICULAR DYSGENESIS SYNDROME: ENVIRONMENTAL CONNECTION"}

\section{To the editor:}

Toppari and co-authors recently published a review on "the environmental connection" of cryptorchidism and hypospadias, including the potential adverse effect of alcohol (Toppari et al., 2010). Based on an article by Damgaard et al., 2007, they conclude that: "mothers' alcohol consumption caused a dose-dependent increase in the risk of cryptorchidism" (Damgaard et al., 2007). We are not convinced that the article by Damgaard and co-authors actually present data that indicate a doseresponse relation, and furthermore, it is not the only paper published on this topic (Jensen et al., 2007; Mongraw-Chaffin et al., 2008; Strandberg-Larsen et al., 2009).

The study by Damgaard et al. (2007) is a follow-up study $(\mathrm{n}=2496)$ with prospective exposure information and clinical assessment of cryptorchidism at birth and at 3 months of age. They report an association between average maternal alcohol consumption during pregnancy and transient cryptorchidism, but this association was apparently driven by an excess risk among few highly exposed boys. Compared with unexposed boys, those exposed to 0.1 to 8.9 drinks per week had an adjusted odds ratio (OR) of cryptorchidism of 0.96 (95\% confidence interval $[\mathrm{CI}], 0.62-1.49)$. Exposure to nine or more and exposure to 9 or more drinks per week (among 5 boys) resulted in an OR of 31.89 (95\% CI, 3.96-256.93). The authors regrouped the exposure categories by consecutively lowering the cut value by one drink per week, and thus admixed an increasing number with lower exposure to the highly exposed open-ended category. For example, boys exposed to five drinks or more per week had an attenuated OR of 3.10 (95\% CI, 1.05-9.10). A true dose-response relation should also be present for the medium exposure groups (e.g., 0.1-4.9 and 0.1-8.9), which was not seen. In fact, the association appears to be driven entirely by few outlying observations, which probably results in an unstable statistical model.

Since Damgaard's study in 2007, three new studies with prospective alcohol exposure classification have been published: two follow-up studies (Jensen et al., 2007; Strandberg-Larsen et al., 2009), and one matched case-control study (Mongraw-Chaffin et al., 2008). None of these studies corroborate an increased risk of cryptorchidism with average weekly alcohol consumption. Two of the studies addressed persisting cryptorchidism and were conducted on older cohorts with higher alcohol exposure contrast than in Damgaard's study (Jensen et al., 2007; Mongraw-Chaffin et al., 2008). Damgaard's findings included transient cryptorchidism, which could explain the incongruences if transient and persisting cryptorchidism do not share this risk factor. This is to our knowledge not supported by experimental or epidemiologic data. There is evidence to indicate that maternal and environmental risk factors do contribute to the occurrence of persisting cryptorchidism (Jensen et al., 2010). A large cohort study $(n=41,268)$ on both maternally reported cryptorchidism (which will include both transient and persisting cases) and persisting cases from treatment reports showed no association with average weekly prenatal alcohol exposure (Strandberg-Larsen et al., 2009). In our view, the combined body of evidence does not support the conclusion by Toppari et al., (2010) on maternal alcohol consumption and cryptorchidism.

\section{REFERENCES}

Damgaard IN, Jensen TK, Petersen JH, et al. 2007. Cryptorchidism and maternal alcohol consumption during pregnancy. Environ Health Perspect 115:272-277.

Jensen MS, Bonde JP, Olsen J. 2007. Prenatal alcohol exposure and cryptorchidism. Acta Paediatr 96:1681-1685.

Jensen MS, Toft G, Thulstrup AM, et al. 2010. Cryptorchidism concordance in monozygotic and dizygotic twin brothers, full brothers, and half brothers. Fertil Steril 93:124-129.

Mongraw-Chaffin ML, Cohn BA, Cohen RD, Christianson RE. 2008. Maternal smoking, alcohol consumption, and caffeine consumption during pregnancy in relation to a son's risk of persistent cryptorchidism: a prospective study in the Child Health and Development Studies cohort, 1959-1967. Am J Epidemiol 167:257-261.

Strandberg-Larsen K, Jensen MS, Ramlau-Hansen CH, et al. 2009. Alcohol binge drinking during pregnancy and cryptorchidism. Hum Reprod 24:3211-3219.

Toppari J, Virtanen H, Main KM, Skakkebaek NE. 2010. Cryptorchidism and hypospadias as a sign of testicular dysgenesis syndrome (TDS): environmental connection. Birth Defects Res A Clin Mol Teratol 88:910-919.

\section{Morten Søndergaard Jensen, ${ }^{1}$ Jens Peter Bonde, ${ }^{2}$ and Jørn Olsen ${ }^{3}$}

${ }^{1}$ Perinatal Research Unit, Department of Pediatrics, Aarhus University Hospital Skejby, Denmark

${ }^{2}$ Department of Occupational and Environmental Medicine, Bispebjerg Hospital, University of Copenhagen, Denmark ${ }^{3}$ Department of Epidemiology, School of Public Health, University of Aarhus, Denmark

Received 21 October 2010; Accepted 29 October 2010

Correspondence to: Morten Søndergaard Jensen (morten@sondergaardjensen.dk)

Published online 2 February 2011 in Wiley Online Library (wileyonlinelibrary. com).

DOI: $10.1002 /$ bdra.20767 